\title{
Down-regulation of the Tumor Suppressor CYLD Enhances the Transformed Phenotype of Human Breast Cancer Cells
}

\author{
TIMOKLIA ORFANIDOU ${ }^{1}$, KONSTANTINOS XANTHOPOULOS ${ }^{1}$, \\ DIMITRA DAFOU ${ }^{1}$, ATHANASIOS PSEFTOGAS ${ }^{1}$, PAUL HADWEH $^{1}$, \\ CLAIRE PSYLLAKI $^{1}$, EUDOXIA HATZIVASSILIOU ${ }^{2}$ and GEORGE MOSIALOS ${ }^{1}$ \\ ${ }^{1}$ School of Biology, ${ }^{2}$ Laboratory of Biological Chemistry, Medical School, \\ Aristotle University of Thessaloniki, Thessaloniki, Greece
}

\begin{abstract}
Background/Aim: The cylindromatosis tumor suppressor (CYLD) has been implicated in the inhibition of human breast cancer development by virtue of the poor prognosis of patients with down-regulated CYLD expression. In order to investigate the mechanism of breast cancer suppression by CYLD, in the present study, cellular and molecular aspects of CYLD-dependent phenotypic regulation of different types of human breast cancer cell lines were analyzed. Materials and Methods: CYLD expression was down-regulated by RNA interference in human breast cancer cell lines. Parental and CYLD-deficient cell lines were evaluated for their viability, migratory capacity, anchorageindependent growth and chemoresistance. Wild-type and mutated forms of CYLD were also evaluated for their ability to suppress the clonogenic potential of breast cancer cells. Results: CYLD down-regulation enhanced the survival and migratory properties of basal and luminal breast cancer cell lines. In addition, down-regulation of CYLD expression enhanced the ability of human breast cancer cells to grow in an anchorage-independent manner and could be associated with resistance to chemotherapeutic drugs. The growthsuppressive properties of CYLD on breast cancer cell lines were dependent on its de-ubiquitinating activity and its amino terminal cytoskeleton-interacting region. Conclusion: Our results establish a broad range of tumor-suppressive properties that are conferred by CYLD in basal and luminal human breast cancer cells and support the significance of
\end{abstract}

This article is freely accessible online.

Correspondence to: George Mosialos, School of Biology, Aristotle University of Thessaloniki, 54124, Thessaloniki, Macedonia, Greece. Tel: +30 2310998907, Fax: +30 2310998907, e-mail: gmosialo@bio.auth.gr

Key Words: CYLD, signal transduction, transformation, tumor suppressor, breast cancer. targeted de-ubiquitination by CYLD in breast cancer cell growth suppression.

The cylindromatosis tumor suppressor gene (CYLD) was originally identified as the defective gene that can predispose humans to the development of skin appendage tumors (1). Subsequent studies indicated that down-regulation of CYLD is associated with the development of several human malignancies that include multiple myeloma, melanoma, colon and hepatocellular carcinoma (reviewed in (2)). A more recent study indicated that CYLD is significantly down-regulated in cancerous compared to normal human breast tissue, thus implicating its function in the homeostasis of human mammary epithelium (3).

Human CYLD encodes a 956-amino acid de-ubiquitinating enzyme (CYLD), which is primarily localized in the cytoplasm. Nuclear localization of CYLD has been also reported although it appears to be transient or tissue and context dependent. The de-ubiquitinating domain of CYLD spans amino acids 589-956 and has specificity for M1- and K63-linked polyubiquitin chains (4). In its amino terminal region CYLD contains three CAP-Gly domains. The two amino-terminal CAP-Gly domains mediate interactions with microtubules (5). The carboxyl-terminal CAPGly domain mediates the interaction of CYLD with Aurora B and the non-catalytic subunit NEMO/IKK $\gamma$ of the IkB-kinase complex $(6,7)$. CYLD affects multiple signaling pathways and cellular processes with significant consequences for cell and tissue homeostasis. It is a negative regulator of several pathways associated with cell survival and proliferation including the NF$\mathrm{kB}, \mathrm{JNK}, \mathrm{p} 38$, Akt, Wnt and TGF $\beta$ pathways (reviewed in (2)). Most of the known regulatory functions of CYLD are associated with its ability to hydrolyze M1- and K63-linked polyubiquitin chains on specific substrates (8). Apparently, these polyubiquitin chains serve as scaffolds onto which signaling molecules are recruited and activated by proximity-induced mechanisms. Notable examples of CYLD targets include members of the TRAF family of signaling molecules, the RIPK1 and TAK1 kinases, NEMO and the NF-kB subunit Bcl3 (9-15). 
A number of evidence supports a role for CYLD in the growth of human breast cancer cells. We have previously shown that exogenous expression of wild-type CYLD, but not a cylindromatosis-associated mutant (CYLD(1-932)) in the aggressive human breast cancer cell line BT549 induces apoptosis and flat cell morphology (16). In addition, it was recently reported that miR-182 sensitizes BT549 cells to TNF $\alpha$-induced apoptosis in a manner that depends on the down-regulation of CYLD by miR-182 (17). Furthermore, a correlation between down-regulation of CYLD by the overexpression of miR182-5p and enhanced oncophenotypic characteristics of human breast cancer MCF7 cells was reported, although it was not demonstrated whether CYLD down-regulation is responsible for the observed phenotype (18). Finally, it has been shown that exogenous expression of CYLD in the MDA-MB-231 breast cancer cell line increased apoptosis and reduced their migratory ability, whereas down-regulation of CYLD in the same cell line has the opposite effects (3).

In the present study we analyzed the growth regulatory effects of CYLD in additional human breast cancer cell lines that expand the range of breast cancer types represented by previously studied cell lines. Furthermore, we have investigated the role of CYLD in additional phenotypic characteristics of human breast cancer cells that include anchorage-independent growth and analyzed the growth suppressive role of specific domains of CYLD. Our findings establish a broad range of tumor-suppressive properties of CYLD towards human breast cancer cells.

\section{Materials and Methods}

Cell culture. The HEK293FT, MDA-MB-231, MCF 7 and HCC1937 cell lines were cultured in Dulbecco's modified Eagle's medium (DMEM; Thermo Fisher Scientific, Waltham, MA, USA) or Roswell Park Memorial Institute (RPMI) 1640 Medium containing $10 \%$ fetal bovine serum (Thermo Fisher Scientific, Waltham, MA, USA), $100 \mathrm{U} / \mathrm{ml}$ penicillin and $100 \mu \mathrm{g} / \mathrm{ml}-$ streptomycin (Thermo Fisher Scientific, Waltham, MA, USA) in a humidified incubator at $37^{\circ} \mathrm{C}$ under $5 \% \mathrm{v} / \mathrm{v} \mathrm{CO}_{2}$.

Lentiviral constructs and transductions. Lentiviral constructs expressing $C Y L D$-targeting (shRNAA, shRNAB) shRNAs were generated by cloning double stranded shRNAA (5'-TGCTGTTGAC AGTGAGCGCGGGAAGTATAGGACAGTATATTAGTGAAGCCA CAGATGTAATATACTGTCCTATACTTCCCTTGCCTACTGCCTC GGA-3'), shRNAB (5'-TGCTGTTGACAGTGAGCGCGGAAGA AGGTCGTGGTCAAGGTAGTGAAGCCACAGATGTACCTTGA CCACGACCTTCTTCCATGCCTACTGCCTCGGA-3') or NSRNA cDNA sequences into the pGIPZ plasmid vector (GE Dharmacon, Lafayette, CO, USA). The non-specific shRNA (NSRNA) was expressed by the non-silencing shRNA mir lentiviral control vector RHS 4346 (GE Dharmacon, Lafayette, CO, USA). The shRNA sequences used were chosen from the NCBI Probe data bank (V2HS_81196 and TRCN0000018366). The double stranded oligonucleotides were cloned into the miR-30 gene sequence between the restriction sites XhoI and EcoRI and the sequences of the cloned oligonucleotides were verified by sequencing. Secondgeneration VSV-G pseudotyped high titers lentiviruses were generated by transient co-transfection of HEK293FT cells without serum $2 \mathrm{hr}$ prior to transfection with a three-plasmid combination as follows: One $100 \mathrm{~mm}$ tissue culture dish containing $3.5 \times 10^{6}$ cells was transfected using $10 \mathrm{mM}$ PEI (Sigma-Aldrich) with Opti-MEM (Thermo Fisher Scientific, Waltham, MA, USA) with $30 \mu \mathrm{g}$ lentiviral vector, $10 \mu \mathrm{g}$ pCMV $\Delta 8.91$ and $20 \mu \mathrm{g}$ pMD VSV-G. Supernatants were collected every 24 and $48 \mathrm{~h}$ after transfection, pulled together and frozen at $-70^{\circ} \mathrm{C}$.

For lentiviral transduction, $1 \times 10^{5}$ cells/well were seeded in 6 well tissue culture plates and infected the following day with shRNAA-, shRNAB- or NSRNA-expressing lentiviruses with $10 \mathrm{mg} / \mathrm{ml}$ Polybrene (Santa Cruz Biotechnology, Heidelberg, Germany). Two days following transduction cells were selected for 20 days with puromycin $(0.3 \mu \mathrm{g} / \mathrm{ml}$ Invivogen, San Diego, CA, USA), and monoclonal cell colonies were subcultured and established.

Quantitative Real Time PCR ( $q P C R$ ). Total cellular RNA was extracted from cells using the Trizol reagent (MRC, Cincinnati, $\mathrm{OH}$, USA). Preservation of $28 \mathrm{~S}$ and $18 \mathrm{~S}$ rRNA species was used to assess RNA integrity. The yield was quantified spectrophoto-metrically. cDNA synthesis was performed using the RevertAid Reverse Transcriptase system (Thermo Fisher Scientific, Waltham, MA, USA) and oligodT18. Analysis of cDNA samples by qPCR was performed using the Applied Biosystems StepOne system and SYBR Green (Sigma-Aldrich) according to the manufacturer's instructions. The PCR program included 1 cycle at $95^{\circ} \mathrm{C}$ for $10 \mathrm{~min}$, and 40 cycles at $95^{\circ} \mathrm{C}$ for $15 \mathrm{~s}$ and at $60^{\circ} \mathrm{C}$ for $1 \mathrm{~min}$. For qPCR the CYLD For (5'-GATTCTGCCTGGCTCTCTTT) and CYLD Rev (5'CAGGTCTTCCAGAGACATCTTC-3') primers were used for the amplification of the corresponding $C Y L D$ mRNA and the YWHAZ For (5'-GCTGGTGATGACCAAGAAAGG-3') and YWHAZ Rev (5'-GGATGTGTTGGTTGCATTTCCT-3') primers were used for the amplification of the corresponding $Y W H A Z$ mRNA. Reactions were done in triplicate using $2 \mu \mathrm{l}$ of cDNA per reaction. Relative expression levels were determined by the $\Delta \Delta \mathrm{CT}$ method.

Immunobloting. Cells were rinsed in cold PBS and whole cell extract was harvested in Nonidet P-40 lysis buffer containing $30 \mathrm{mM}$ Tris ( $\mathrm{pH}$ 7.5), $150 \mathrm{mM} \mathrm{NaCl}, 10 \%$ glycerol, $1 \%$ Nonidet P-40, and a cocktail of protease inhibitors (Sigma-Aldrich). Whole cell lysates were homogenized and following centrifugation $(13,000 \times g$ for $15 \mathrm{~min})$ to remove cell debris, the extract was boiled in loading buffer containing $1 \%$ SDS, 0.1M DTT. Extracted proteins were quantified by the Bradford assay and $25 \mu \mathrm{g}$ of protein/lane was separated by SDS-PAGE electrophoresis and transferred to a Hybond-ECL nitrocellulose membrane (Amersham Biosciences, Piscataway, NJ, USA). Blots were incubated at room temperature for $1 \mathrm{~h}$ in 1:200 v/v anti-CYLD E-4 (Santa Cruz Biotechnology, Heidelberg, Germany), 1:1000 v/v anti- $\beta$ actin (Santa Cruz Biotechnology, Heidelberg, Germany), followed by $1 \mathrm{~h}$ incubation at room temperature in 1:2000 anti-mouse-horseradish peroxidase-conjugated antibody. Bands were visualized by ECLchemiluminesence and quantified by ImageJ.

Cell viability assay. Cell viability assay was performed using the 3-(4,5-dimethylthiazol-2-yl)-2,5-diphenyltetrazolium bromide (MTT) (Sigma-Aldrich) dye reduction method. MDA-MB-231, MCF-7 and HCC1937 NSRNA and shRNA stable lines were seeded 
A

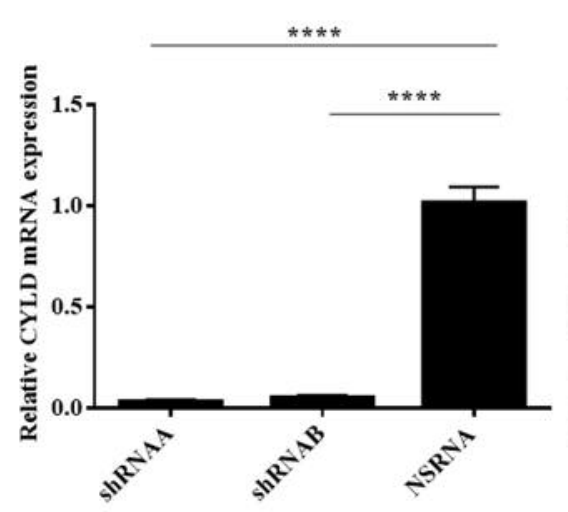

MCF-7

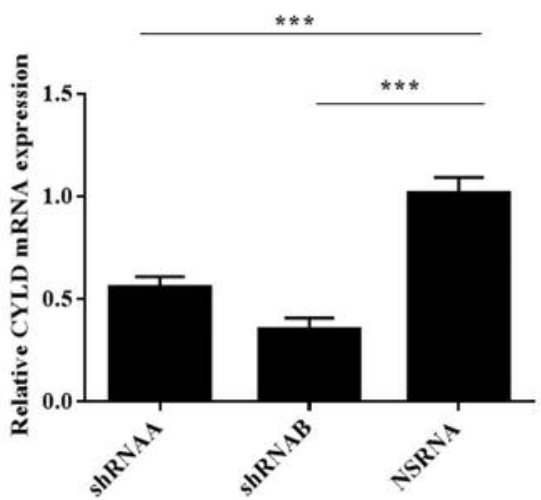

HCC1937

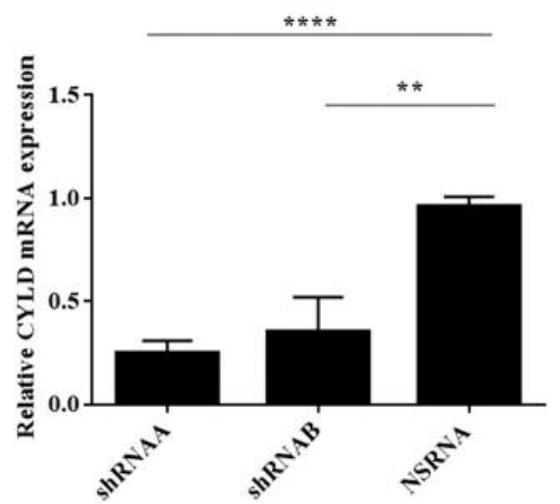

B

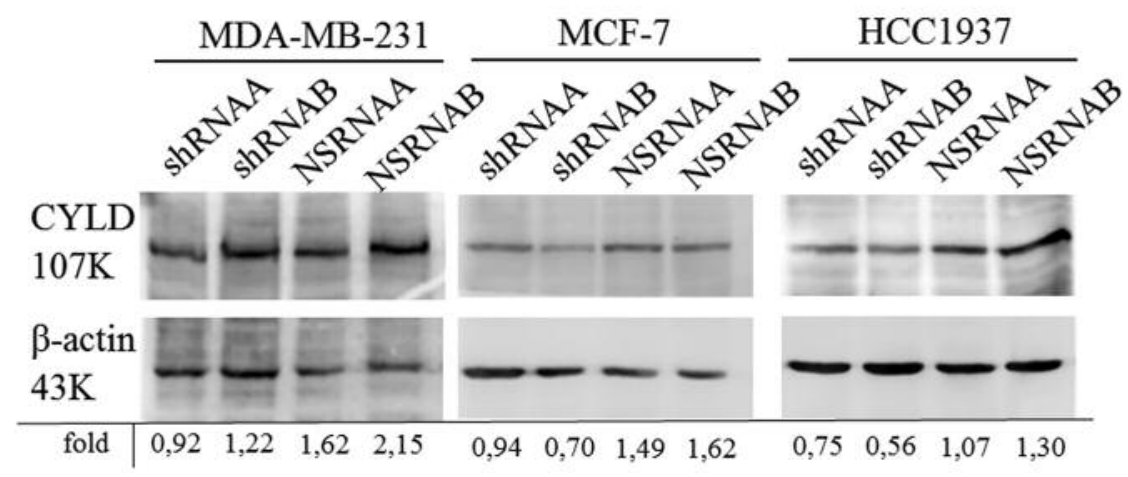

Figure 1. Down-regulation of CYLD expression by RNA interference in breast cancer cell lines. CYLD mRNA (A) and protein (B) expression in MDA-MB-231, MCF-7 and HCC1937 clones stably transduced with lentiviruses expressing CYLD-targeting shRNAs (shRNAA, shRNAB) or a nonspecific shRNA (NSRNAA, NSRNAB). YWHAZ was used as a normalization control gene for 9 PCR analysis in A and $\beta$-actin as a loading control in immunoblot analysis in B. Data in A are presented as the mean values ( \pm SEM) from three independent experiments. Statistical evaluation of the differences between the values indicated by horizontal lines was performed by Student's t-test. Statistical significance is indicated by two ( $p<0.01$ ), three $(p<0.001)$ or four $(p<0.0001)$ stars. Representative immunoblots are shown in B. The values below each lane indicate the ratio of the CYLD band intensity to the corresponding intensity of the $\beta$-actin band as determined by the Image $J$ software.

at a density of $5 \times 10^{3}$ cells per well in 96 well flat bottom culture plates and allowed to attach and grow overnight at $37 \mathrm{oC}$ with $5 \%$ $\mathrm{v} / \mathrm{v} \mathrm{CO}_{2}$ in a cell culture incubator. The next day the cells were serum starved overnight and $24 \mathrm{~h}$ later MTT was added to a final concentration of $0.45 \mathrm{mg} / \mathrm{ml}$ in each well (final volume $220 \mu \mathrm{l}$ ) and the plate was incubated for $4 \mathrm{~h}$ at $37^{\circ} \mathrm{C}$ with $5 \% \mathrm{v} / \mathrm{v} \mathrm{CO}$ level. To dissolve insoluble formazan crystals formed in mitochondria, 150 $\mu \mathrm{l}$ of acid isopropanol containing 10\% Triton-X (Applichem, Darmstadt, Germany) were added to each well and the plate was incubated with gentle shaking for $5 \mathrm{~min}$. The resulting color intensity was measured at 570 and $630 \mathrm{~nm}$ absorbance using a Microplate Autoreader (Biotek, Winooski, VT, USA). Each sample was tested in triplicates and experiments were repeated twice.

Anchorage-independent proliferation assay. Three milliliters of $0.33 \%$ "top" agar containing $5 \times 10^{3}$ cells were plated over a $0.66 \%$ "bottom" agar layer. All samples were plated in triplicate and three independent experiments were performed to test for statistical significance. Cells were incubated at $37^{\circ} \mathrm{C}$ for 3-4 weeks before fixing/staining with $1 \mathrm{mg} / \mathrm{ml} \mathrm{p}$-iodonitrotetrazolium violet (SigmaAldrich) in methanol overnight at $37^{\circ} \mathrm{C}$ in a humidified environment. Red-stained colonies with more than 30 cells were counted. Images were taken at $1 \mathrm{x}$ and $6 \mathrm{x}$ zoom.

Migration assay. For in vitro cell migration assay, 104 cells were plated in an Ibidi tissue culture insert with serum-free media. The insert was removed $24 \mathrm{~h}$ later and migration of cells was assessed by measuring the cell-free gap width using a Axiovert $40 \mathrm{C}$ microscope (Carl Zeiss, AG, Oberkochen, Germany). Images were analyzed with the Image J software.

Clonogenic assay. Plasmids expressing FLAG-tagged (F-) CYLD, CYLDC601S, CYLD(538-953), were previously described (10). An expression plasmid of FLAG-tagged CYLD with phenylalanine codons 518 and 533 mutated to alanine codons (F-CYLDF518A/ F533A) was constructed by site directed mutagenesis. The 
A

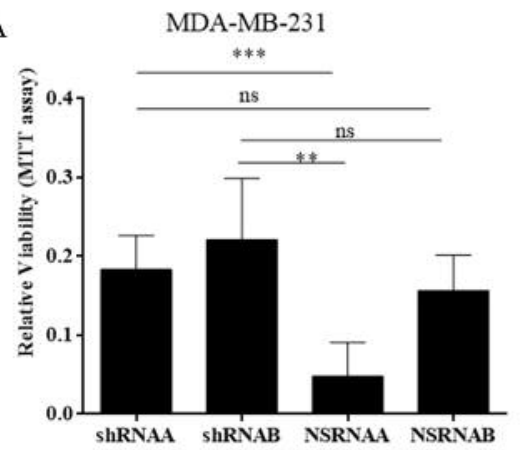

B

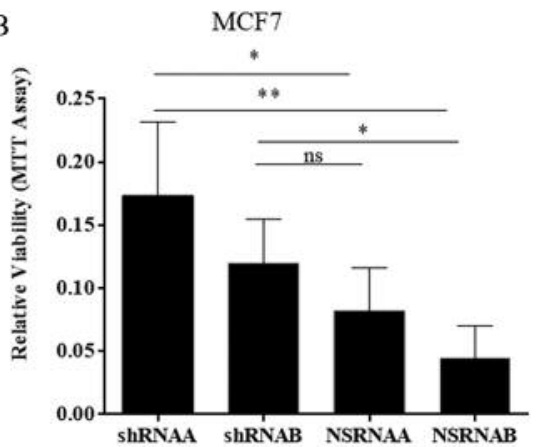

$\mathrm{C}$

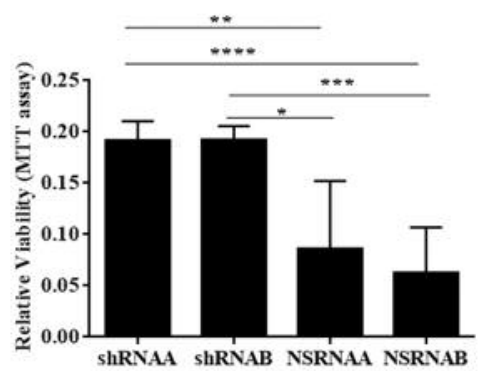

Figure 2. Down-regulation of CYLD expression can increase the viability of breast cancer cell lines. The relative viability of MDA-MB-231 (A), MCF-7 (B) and HCC1937 (C) clones transduced with lentiviruses expressing CYLD-targeting shRNAs (shRNAA, shRNAB) or a non-specific shRNA (NSRNAA, $N S R N A B)$ and cultured in serum-free medium was determined by the MTT assay. Values are mean $\pm S E M$ of triplicate samples. Statistical evaluation of the differences between the values indicated by horizontal lines was performed by Student's t-test. ${ }^{*} p<0.05, * * p<0.01, * * * p<0.001, * * * * p<0.0001, n s p>0.05$.

expression plasmids were used to transfect the MDA-MB-231 cells and establish G418-resistant colonies. The transfection procedure was performed using calcium chloride (19) and the efficiency was evaluated by western blotting analysis. At the sametime, MDA-MB231 cells were transfected seeded in 6-well-plates at a density of 1000 cells per well and cultured for 21 days in the presence of 0.5 $\mathrm{mg} / \mathrm{ml} \mathrm{G} 418$ (Invivogen, San Diego, CA, USA). Then, the colonies were fixed with methanol for $10 \mathrm{~min}$ and stained with crystal violet for $15 \mathrm{~min}$, after which point the number of colonies containing more than 50 cells was scored. Relative colony formation efficiency was calculated by dividing the number of colonies by the relative intensity of the corresponding CYLD to actin immunoblot band and the number of colonies generated by the transfection of the empty pCDNA3 vector.

Chemoresistance assays. HCC1937 clones stably transduced with CYLD-targeting shRNA- or NSRNA-expressing vectors were seeded at a density of $1 \times 10^{4}$ cells per well in 96-well flat bottom culture plates and allowed to attach and grow overnight in a humidified incubator at $37^{\circ} \mathrm{C}$ under $5 \% \mathrm{v} / \mathrm{v} \mathrm{CO}_{2}$. The next day the cells were treated with Doxorubicin $(2 \mu \mathrm{M})$, Etoposide $(200 \mu \mathrm{M})$ or DMSO $\left(0.5 \% \mathrm{v} / \mathrm{v}\right.$, control) for $72 \mathrm{~h}$ at $37^{\circ} \mathrm{C}$ with $5 \% \mathrm{v} / \mathrm{v} \mathrm{CO} 2$ level. Cell viability was estimated with the MTT assay as previously described.

Statistical analysis. All experiments were performed in triplicate and analysed by unpaired $t$-test using a confidence level of $95 \%$ $(p<0.05)$. Numerical data were expressed as mean \pm Standard Deviation (SD) or mean \pm Standard Error of the Mean (SEM). Statistical analysis was performed using the SPSS software (version 22.0) and GraphPad Prism (version 6.01).

\section{Results}

Down-regulation of CYLD augments the viability, migratory ability and anchorage-independent growth of breast cancer cell lines. In order to evaluate the role of CYLD in the growth properties of different subtypes of breast cancer, its expression was down-regulated by RNA interference in cell lines representing basal (MDA-MB-231, HCC1937) and luminal (MCF7) types of breast cancer (20). For each cell line two clones transduced with either shRNAA or shRNAB and two clones transduced with NSRNA (NSRNAA and NSRNAB) were isolated. The down-regulation of CYLD mRNA and protein expression in the clones that were transduced with either shRNAA or shRNAB was verified by qPCR (Figure 1A) and immunoblotting (Figure 1B), respectively. RNA interference caused a greater reduction in the level of $C Y L D$ mRNA compared to the reduction in CYLD protein levels. This is consistent with the low levels of CYLD protein and the small range of CYLD protein fluctuation in most epithelial cells. To determine whether CYLD down-regulation affected the viability of breast cancer cell lines, the clones that were transduced with shRNA-expressing vectors were evaluated for their viability following $24 \mathrm{~h}$ of serum starvation. As shown in Figure 2, cell lines with down-regulated CYLD expression in general exhibited higher viability upon serum starvation compared to the corresponding cell lines that were transduced with control shRNA-expressing vectors.

The ability of breast cancer cells to metastasize can be monitored, in part, by evaluating their migratory capacity on a solid substrate. In order to determine whether CYLD expression levels affect the ability of breast cancer cells to migrate, MDA-MB-231, HCC1937 and MCF7 cell lines with down-regulated CYLD expression were tested for their migratory capacity using a standard cell migration assay, as described in the materials and methods section. Downregulation of CYLD increased significantly the migratory capacity of all cell lines, as documented by the faster closure of the cell culture gap in clones with reduced expression of CYLD, compared to the corresponding control cell line clones (Figure 3).

A characteristic feature of aggressive cancer cells is their ability to grow in an anchorage-independent manner. In order 

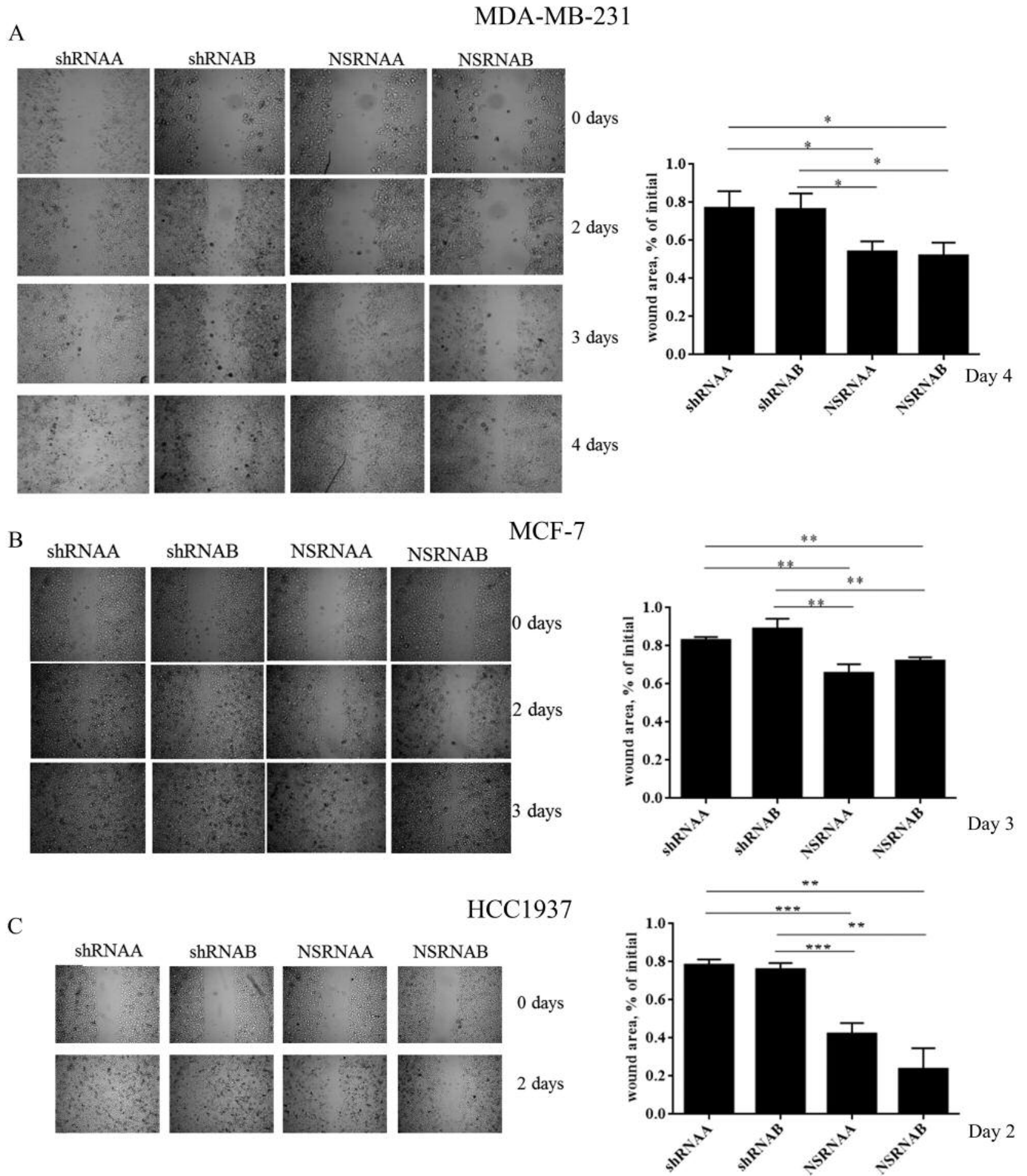

Figure 3. Down-regulation of CYLD expression can increase the migration capacity of breast cancer cell lines. Migration capacity evaluation of MDA-MB-231 (A), MCF-7 (B) and HCC1937 (C) clones transduced with lentiviruses expressing CYLD-targeting shRNAs (shRNAA, shRNAB) or a non-specific shRNA (NSRNAA, NSRNAB). Cells were seeded in ibidi's culture inserts. The next day the inserts were removed and migration of cells was assessed by measuring the cell-free gap size at three positions along the gap and averaging the values. Representative pictures of the cell cultures taken at the indicated time points after removal of the insert are shown on the left side of each section. The histograms indicate mean values $( \pm S E M)$ of cell-free gap sizes expressed as percent of initial gap size from three independent experiments. Statistical evaluation of the differences between the values indicated by horizontal lines was performed by the Student's t-test. ${ }^{*} p<0.05, * * p<0.01, * * * p<0.001$. 
A

MDA-MB-231

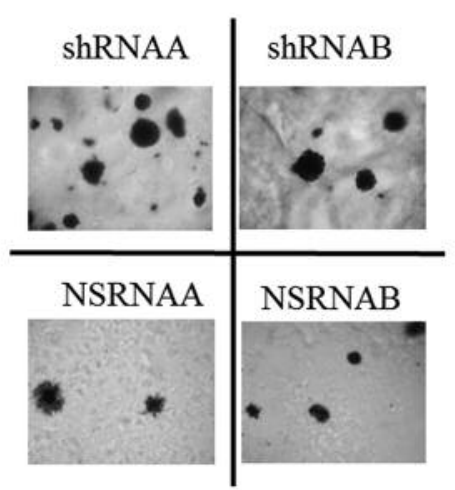

6x camera zoom

B

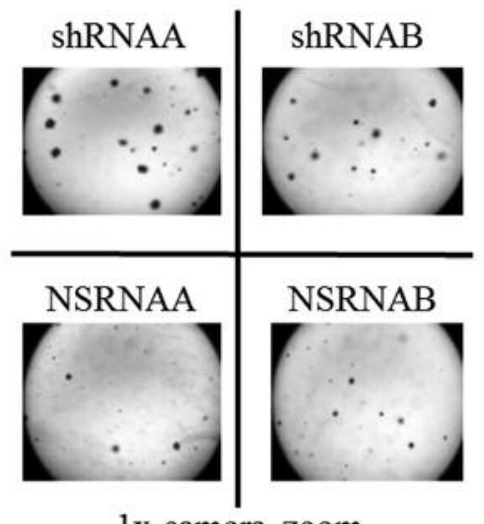

1x camera zoom

C

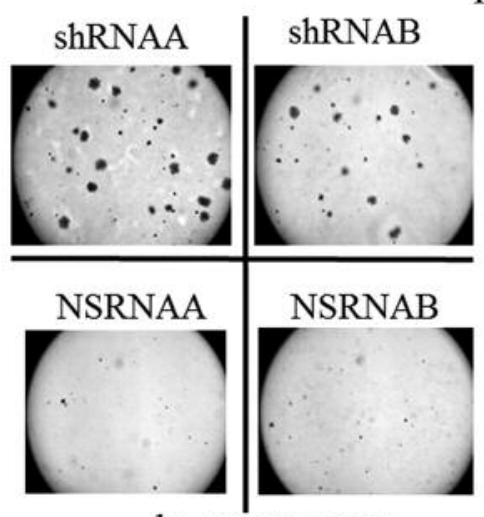

1x camera zoom

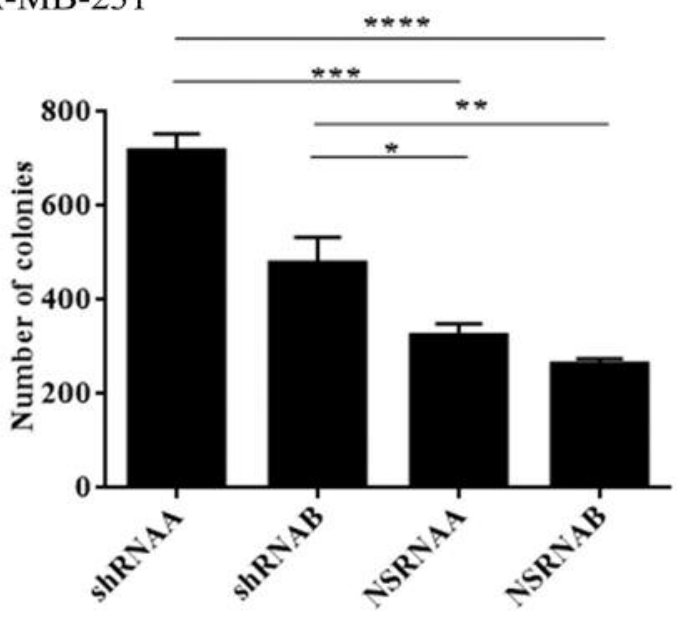

MCF-7

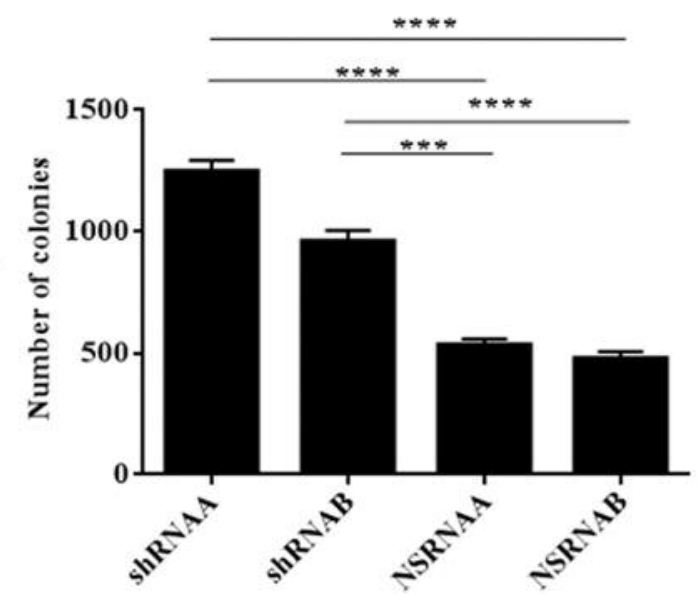

HCC 1937

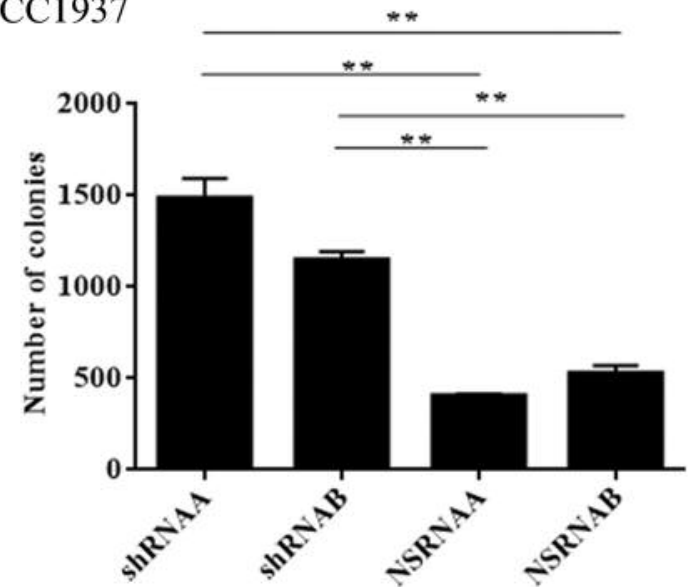

Figure 4. Down-regulation of CYLD expression can enhance the anchorage-independent growth capacity of breast cancer cell lines. Anchorageindependent growth capacity of MDA-MB-231 (A), MCF-7 (B) and HCC1937 (C) clones transduced with lentiviruses expressing CYLD-targeting shRNAs (shRNAA, shRNAB) or a non-specific shRNA (NSRNAA, NSRNAB). Representative pictures of cell cultures at the time of scoring are shown on the left side of each section. The histograms indicate mean values $( \pm S E M)$ of colonies grown in soft agar from three independent experiments. Statistical evaluation of the differences between the values indicated by horizontal lines was performed by the Student's $t$-test. ${ }^{*} p<0.05$, **p<0.01, $* * * p<0.001, * * * * p<0.0001$. 
A

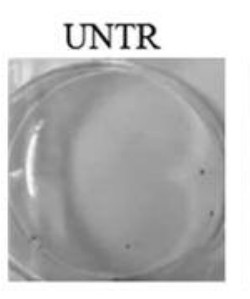

B
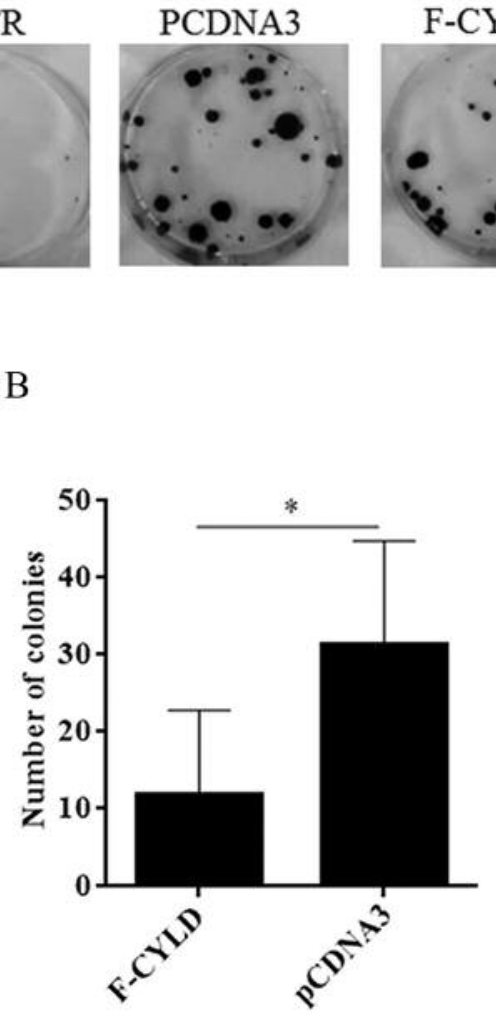
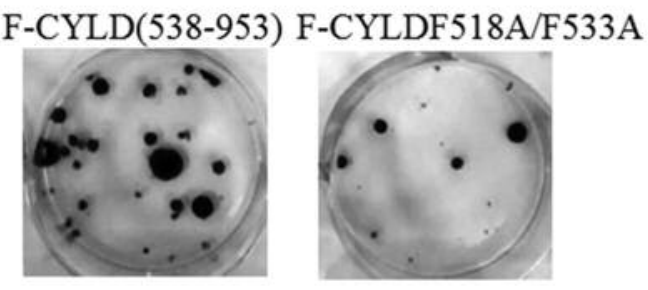

F-CYLD

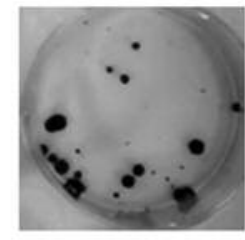

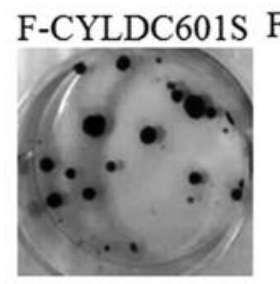

$\mathrm{C}$

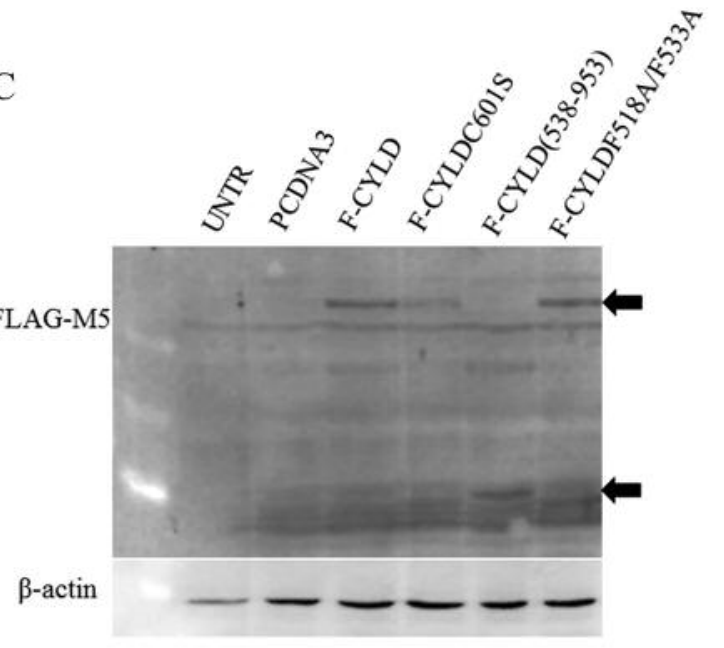

$\mathrm{D}$

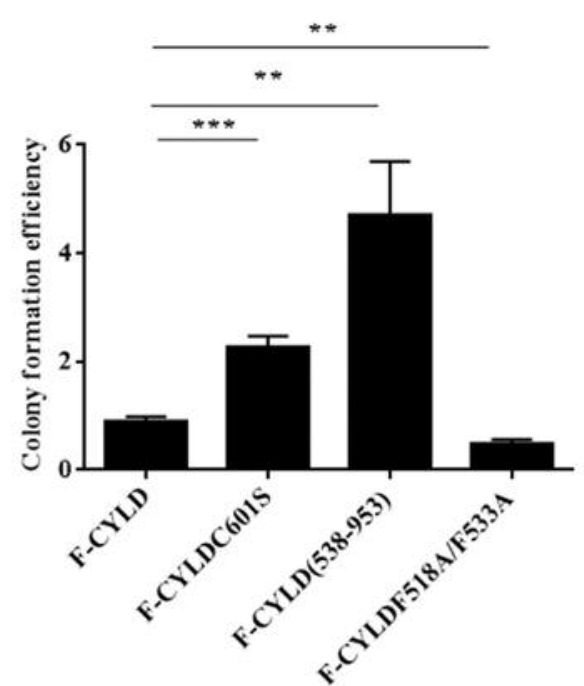

Figure 5. Clonogenic assay to evaluate the effect of wild-type and mutated forms of CYLD. MDA-MB-231 cells were transfected with plasmids expressing F-CYLD, F-CYLDC601S, F-CYLD(538-953), F-CYLDF518A/F533A. 48h post-transfection $10^{3}$ cells were counted, seeded in 6-well-plates and cultured for 21 days in the presence of $0.5 \mathrm{mg} / \mathrm{ml} \mathrm{G418.} \mathrm{The} \mathrm{number} \mathrm{of} \mathrm{colonies} \mathrm{containing} \mathrm{more} \mathrm{than} 50$ cells was scored. (A) Representative pictures of cell clones that were scored. (B) Histogram of colony formation efficiency of MDA-MB-231 cells transfected with $p C D N A 3$ vector or a F-CYLD-expressing vector. Mean values of colony numbers $( \pm$ SEM) from three independent experiments are shown. Statistical evaluation was performed by Student's $t$-test and the star indicates $p<0.05$. (C) Evaluation of expression of FLAG-tagged proteins in MDA-MB-231 cells 48 h post-transfection by immunoblotting using an anti-FLAG monoclonal antibody. (D) Histogram of the relative colony formation efficiencies that correspond to the transfection of plasmids that express the indicated proteins. Values represent the mean relative colony formation efficiencies $( \pm S E M)$ from three independent experiments. Statistical evaluation of the differences between the values indicated by horizontal lines was performed by the Student's $t$-test. * $p<0.05$, ${ }^{* *} p<0.01,{ }^{* * *} p<0.001$. 


\section{MDA-MB-231}

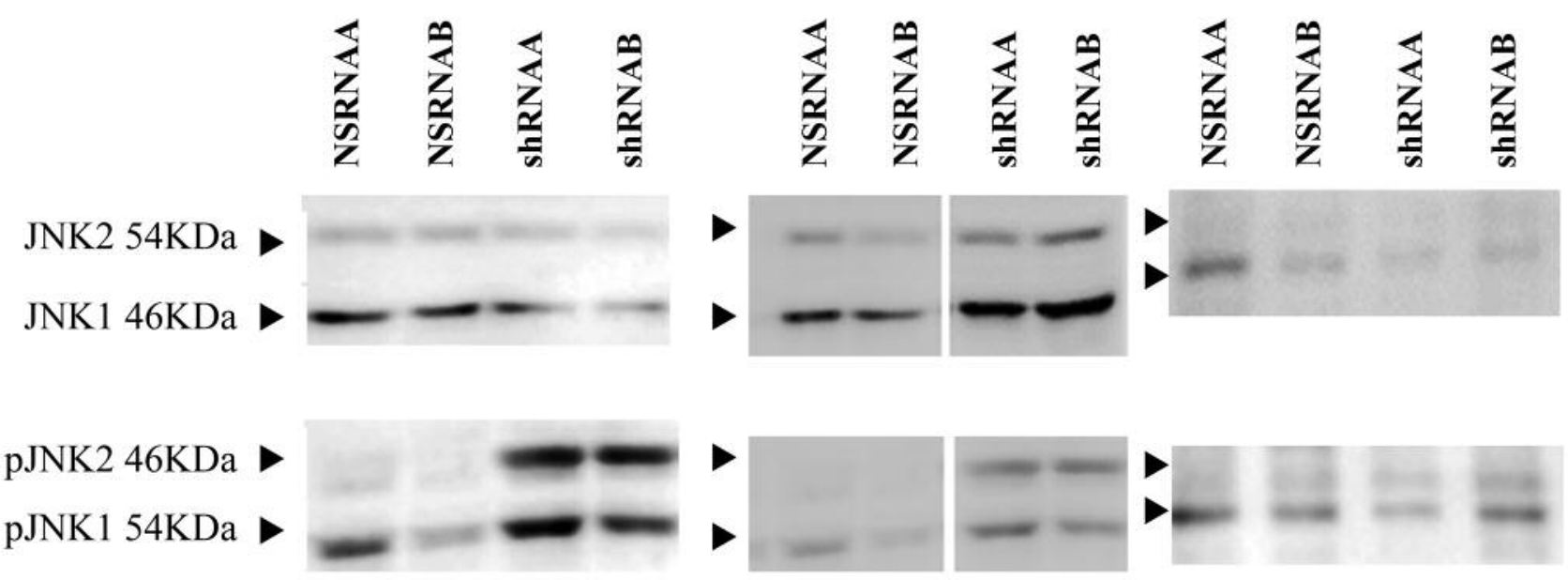

Figure 6. Effect of CYLD down-regulation on the basal activities of JNK in breast cancer cells lines. Whole-cell extracts of MDA-MB-231, MCF-7 and HCC1937 clones stably transduced with lentiviruses expressing CYLD-targeting shRNAAs (shRNA, shRNAB) or a non-specific shRNA (NSRNAA, $N S R N A B)$ were analyzed by immunoblotting with antibodies that recognize total (JNK1, JNK2) or phosphorylated (pJNK1, pJNK2) JNK1 and JNK2.

to determine whether CYLD can affect the anchorageindependent growth capacity of breast cancer cells, MDAMB-231, HCC1937 and MCF7 cell lines with downregulated CYLD expression were tested for their ability to form colonies in soft agar. In all three cell lines tested, CYLD down-regulation caused dramatic increase in the number of colonies that could grow in soft agar (Figure 4). These results corroborate the breast cancer suppressive role of CYLD, which has been highlighted by the survival and migration assays presented above.

The de-ubiquitinating domain and cytoskeleton-interacting region of CYLD play an important role in the growth suppression of breast cancer cell lines. The molecular mechanism underlying CYLD-mediated growth suppression of breast cancer cells is poorly understood. In order to identify functional properties of CYLD that are important for the growth suppression of breast cancer cells, the ability of wild-type and mutant isoforms of CYLD to suppress colony formation in MDA-MB-231 cells was evaluated. The mutations that were tested are known to affect specific functional domains or regions of CYLD. The FCYLDC601S mutant contains a single cysteine to serine codon alteration at position 601 that inactivates the deubiquitinating activity of CYLD. The F-CYLDF518A/F533A mutant contains two phenylalanine to alanine codon alterations at positions 518 and 533, which are located at the interface of CYLD-NEMO interacting domains. F-
CYLDF518A/F533A is significantly compromised in its ability to suppress TRAF2-mediated NF-kB activation compared to wild-type CYLD (data not shown). The FCYLD(538-953) mutant lacks the amino terminal amino acids 1-537, which include the three CAP-Gly domains, but contains the entire catalytic region of CYLD. Wild-type CYLD was capable of dramatically suppressing the ability of MDA-MB-231 to form colonies on a solid substrate (Figure 5). F-CYLDC601S and F-CYLD(538-953) were significantly compromised in their ability to suppress the clonogenic capacity of MDA-MB-231 compared to wildtype CYLD. Finally, F-CYLDF518A/F533A suppressed the clonogenic capacity of MDA-MB-231 similarly to wild-type CYLD. These results indicate that the de-ubiquitinating activity of CYLD is essential, but not sufficient to suppress the growth of breast cancer cells. Furthermore, the amino terminal region of CYLD, which contains the three CAP-Gly domains, is also important for the growth suppression of breast cancer cells by CYLD. Finally, the NEMO-interacting domain of CYLD does not appear to play an essential role in the suppression of breast cancer cell growth (Figure 5).

CYLD can suppress the activity of JNK in breast cancer cell lines. It has been shown in various cell types that CYLD can negatively regulate the activation of JNK and the NF-kB transcription factor. In order to determine whether CYLD can inhibit the basal activity of these pathways in breast cancer cell lines, the relative levels of phosphorylated JNK were 


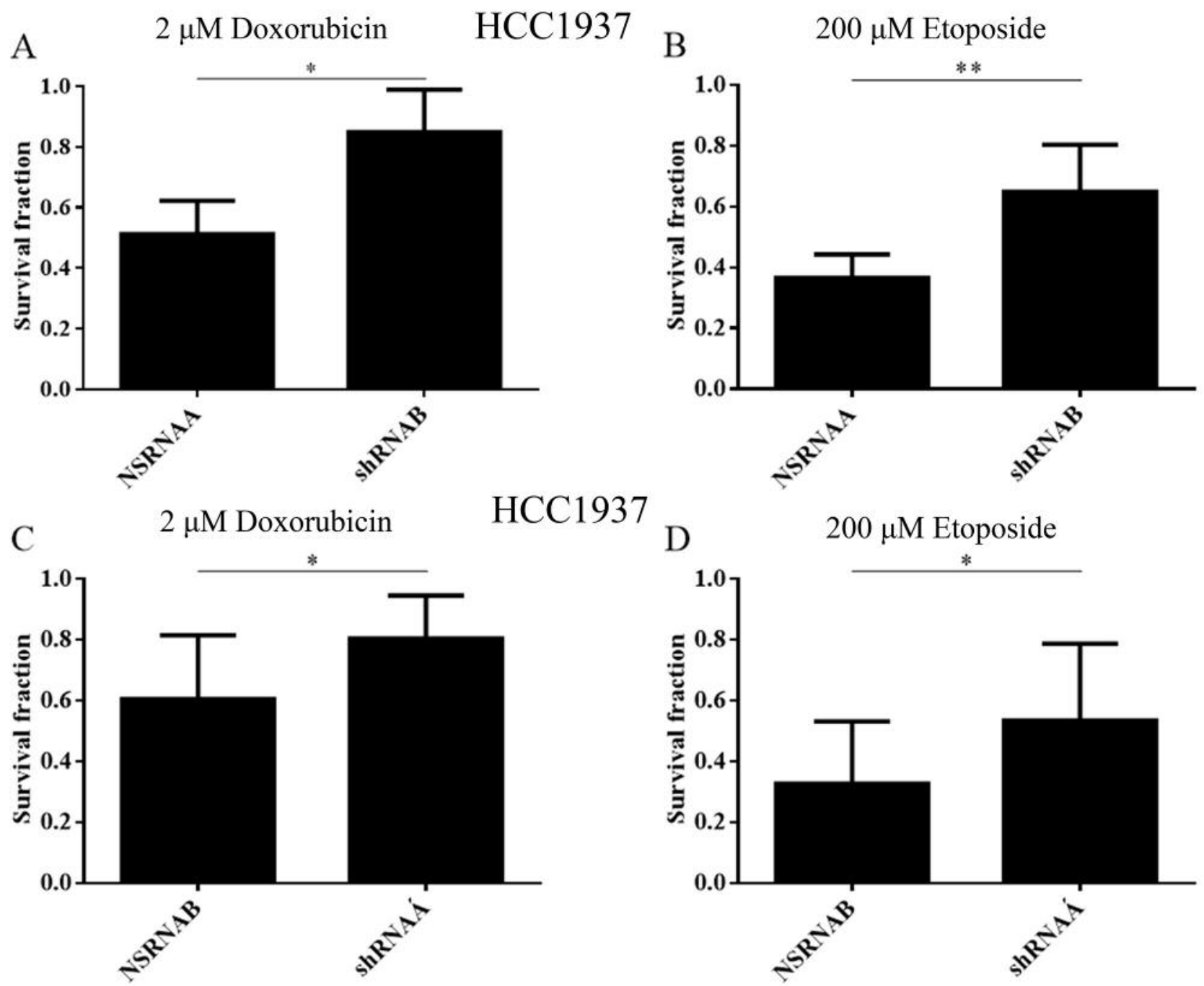

Figure 7. Effect of CYLD down-regulation on the chemoresistance of breast cancer cell lines. HCC1937 clones stably transduced with lentiviruses expressing CYLD-targeting shRNAs (shRNAA, shRNAB) or a non-specific shRNA (NSRNAA, NSRNAB) were treated with doxorubicin $2 \mu M(A, C)$, etoposide $200 \mu M(B, D)$ or DMSO (control) for $72 \mathrm{~h}$ and then cell viability was assessed with the MTT assay. Survival fraction was estimated as the fraction of viable cells in the presence of the agent vs. viable cells in the presence of DMSO. Each experiment was performed in technical triplicates and a minimum of three biological replicates were performed. Columns indicate the mean of the replicates $\pm S D$. $* 0.01<p<0.05$ and $* * 0.001<p<0.01$ (one-tailed Student's t-test).

determined in CYLD-deficient and control breast cancer cell lines. Down-regulation of CYLD resulted in significant enhancement of JNK1 and JNK2 phosphorylation in MDAMB-231 cells and JNK2 phosphorylation in MCF7 cells. On the other hand, down-regulation of CYLD in HCC1937 cells did not result in enhanced activation of JNK (Figure 6). Finally, down-regulation of CYLD did not augment the phosphorylation of $\mathrm{p} 105 / \mathrm{NF}-\mathrm{kB} 1$ in HCC1937 (data not shown). These results indicate that CYLD can down-regulate the basal activity of JNK at least in a subset of breast cancer cell lines whereas basal NF-kB activity does not appear to be regulated by CYLD in breast cancer cell lines universally.
The role of CYLD in breast cancer cell line chemosensitivity. Down-regulation of CYLD expression has been associated with the resistance of breast cancer to chemotherapeutic agents in patients (3). In order to determine whether down-regulation of CYLD can confer chemoresistance to breast cancer cells the effect of chemotherapeutic agents doxorubicin and etoposide on the survival of control and CYLD-deficient HCC1937 cells was evaluated. These experiments showed that down-regulation of CYLD can increase significantly the resistance of HCC1937, but not MDA-MB-231 or MCF7 cells to doxorubicin or etoposide treatment (Figure 7, data not shown). It should be noted however, that transient down- 
regulation of CYLD expression by siRNA transfection, did not increase significantly the resistance of HCC1937 cells to doxorubicin or etoposide, possibly due to the inability of the cells to adjust to acute CYLD deficiency. Our findings suggest that CYLD down-regulation can contribute to chemoresistance of a subset of breast cancer cells, possibly by cooperating with other genetic or epigenetic alterations.

\section{Discussion}

Down-regulation of CYLD expression has been correlated with the development of various types of human malignancies (2). However, the causative role of CYLD down-regulation in the deregulation of homeostatic mechanisms of various cell types that lead to transformation has not been established. Previous studies have indicated that increased expression of CYLD in two basal-type breast cancer cell lines (BT-549 and MDA-MB-231) promotes their apoptosis whereas down-regulation of CYLD in MDA-MB231 inhibits apoptosis and promotes cell migration $(3,16$, 17). In the present study we demonstrated for the first time, that the direct growth and migratory-suppressive role of CYLD is also evident in a luminal type breast cancer cell line (MCF7) and an additional basal type breast cancer cell line (HCC1937). Most importantly we have demonstrated for the first time, that CYLD down-regulation enhances the ability of basal and luminal types of breast cancer cells to grow in an anchorage independent manner. Anchorageindependent growth is a hallmark of aggressive cancer cells and our findings are consistent with the correlation of patient poor prognosis with down-regulated CYLD expression (3).

The functional characterization of CYLD domains indicated a critical role for the de-ubiquitinating activity of CYLD in the growth suppression of breast cancer cells. This finding suggests that increased ubiquitination of a specific substrate plays a critical role in promoting the survival of breast cancer cells. Previous studies have demonstrated that CYLD can mediate the removal of K63- and M1-linked polyubiquitin chains from various molecules that can orchestrate the activation of NF-kB and MAP kinase pathways. Under steady state conditions of cell culture we were unable to document increased ubiquitination of several known targets of CYLD that include RIP1, TRAF2, TRAF6 and NEMO in HCC1937 breast cancer cells with reduced CYLD expression (data not shown). It is possible that CYLD regulates the ubiquitination of one of these molecules in breast cancer cells upon stimulation by certain ligands or in a cell-line-dependent manner. Alternatively, the molecule or molecules whose CYLD-dependent ubiquitination regulate the growth of breast cancer cells is not among the aforementioned molecules and remains elusive. Interestingly, the tumor suppressing function of CYLD was associated with the regulation of JNK activity in specific breast cancer cell lines for the first time, suggesting a possible role of CYLD-dependent JNK activation in the enhancement of the oncophenotypic characteristics of specific breast cancer cells.

In the present study we demonstrated for the first time that although the de-ubiquitinating activity of CYLD is essential for the growth suppression of breast cancer cells it is clearly not sufficient, since a deubiquitinase proficient CYLD molecule that lacks the amino terminal 540 amino acids was unable to suppress the growth of breast cancer cells. This result indicates that the proper localization and/or protein-interactions of CYLD are required in addition to its de-ubiquitinating activity in order to suppress the growth of breast cancer cells. The amino terminal CAP-Gly domain of CYLD mediates its interaction with microtubules and this interaction may be essential for targeting specific substrates for deubiquitination in order to suppress the growth of breast cancer cells.

The effect of CYLD down-regulation on chemoresistance was evaluated on all available stably-transduced breast cancer cell lines, both of the basal- and the luminal-type. However, differences in chemoresistance were only observed in HCC1937 cells. This difference in response could be associated with the higher basal levels of CYLD in this cell line, rather than the cell line type (basal or luminal). Further correlation studies of CYLD expression levels and chemoresistance could lead to establishment of CYLD as a prognostic marker for treatment efficacy at least in a subset of breast cancer cases.

\section{Conclusion}

In summary, our results establish the tumor-suppressive role of CYLD in different types of breast cancer cells and associate the tumor-suppressing function of CYLD with its ability to mediate targeted de-ubiquitination and deregulation of JNK activity. Our findings support the possibility of incorporating the molecular characterization of CYLD in prognostic and predictive protocols for breast cancer.

\section{Funding}

This research has been co-financed by the European Union (European Social Fund - ESF) and Greek national funds through the Operational Program "Education and Lifelong Learning" of the National Strategic Reference Framework (NSRF) - Research Funding Program: ARISTEIA-1921-EMBRACE. Investing in knowledge society through the European Social Fund.

\section{References}

1 Bignell GR, Warren W, Seal S, Takahashi M, Rapley E, Barfoot R, Green H, Brown C, Biggs PJ, Lakhani SR, Jones C, Hansen J, Blair E, Hofmann B, Siebert R, Turner G, Evans DG, Schrander-Stumpel C, Beemer FA, van Den Ouweland A, Halley D, Delpech B, Cleveland MG, Leigh I, Leisti J and Rasmussen S: Identification of the familial cylindromatosis tumoursuppressor gene. Nat Genet 25: 160-165, 2000. 
2 Mathis BJ, Lai Y, Qu C, Janicki JS and Cui T: CYLD-mediated signaling and diseases. Curr Drug Targets 16: 284-294, 2015.

3 Hayashi M, Jono H, Shinriki S, Nakamura T, Guo J, Sueta A, Tomiguchi M, Fujiwara S, Yamamoto-Ibusuki M, Murakami K, Yamashita S, Yamamoto Y, Li JD, Iwase H and Ando Y: Clinical significance of CYLD down-regulation in breast cancer. Breast Cancer Res Treat 143: 447-457, 2014.

4 Komander D, Lord CJ, Scheel H, Swift S, Hofmann K, Ashworth A and Barford D: The structure of the CYLD USP domain explains its specificity for Lys63-linked polyubiquitin and reveals a B box module. Mol Cell 29: 451-464, 2008.

5 Gao J, Huo L, Sun X, Liu M, Li D, Dong JT and Zhou J: The tumor suppressor CYLD regulates microtubule dynamics and plays a role in cell migration. J Biol Chem 283: 8802-8809, 2008.

6 Sun L, Gao J, Huo L, Sun X, Shi X, Liu M, Li D, Zhang C and Zhou J: Tumour suppressor CYLD is a negative regulator of the mitotic kinase Aurora-B. J Pathol 221: 425-432, 2010.

7 Saito K, Kigawa T, Koshiba S, Sato K, Matsuo Y, Sakamoto A, Takagi T, Shirouzu M, Yabuki T, Nunokawa E, Seki E, Matsuda T, Aoki M, Miyata Y, Hirakawa N, Inoue M, Terada T, Nagase T, Kikuno R, Nakayama M, Ohara O, Tanaka A and Yokoyama S: The CAP-Gly domain of CYLD associates with the prolinerich sequence in NEMO/IKKgamma. Structure 12: 1719-1728, 2004.

8 Komander D, Reyes-Turcu F, Licchesi JD, Odenwaelder P, Wilkinson KD and Barford D: Molecular discrimination of structurally equivalent Lys 63-linked and linear polyubiquitin chains. EMBO Rep 10: 466-473, 2009.

9 Reiley W, Zhang M, Wu X, Granger E and Sun SC: Regulation of the deubiquitinating enzyme CYLD by IkappaB kinase gamma-dependent phosphorylation. Mol Cell Biol 25: 38863895, 2005.

10 Trompouki E, Hatzivassiliou E, Tsichritzis T, Farmer $\mathrm{H}$, Ashworth A and Mosialos G: CYLD is a deubiquitinating enzyme that negatively regulates NF-kappaB activation by TNFR family members. Nature 424: 793-796, 2003.

11 Brummelkamp TR, Nijman SM, Dirac AM and Bernards R: Loss of the cylindromatosis tumour suppressor inhibits apoptosis by activating NF-kappaB. Nature 424: 797-801, 2003.

12 Kovalenko A, Chable-Bessia C, Cantarella G, Israel A, Wallach $\mathrm{D}$ and Courtois G: The tumour suppressor CYLD negatively regulates NF-kappaB signalling by deubiquitination. Nature 424 : 801-805, 2003.
13 Wright A, Reiley WW, Chang M, Jin W, Lee AJ, Zhang M and Sun SC: Regulation of early wave of germ cell apoptosis and spermatogenesis by deubiquitinating enzyme CYLD. Dev Cell 13: 705-716, 2007.

14 Massoumi R, Chmielarska K, Hennecke K, Pfeifer A and Fassler R: Cyld inhibits tumor cell proliferation by blocking Bcl-3dependent NF-kappaB signaling. Cell 125: 665-677, 2006.

15 Reiley WW, Jin W, Lee AJ, Wright A, Wu X, Tewalt EF, Leonard TO, Norbury CC, Fitzpatrick L, Zhang M and Sun SC: Deubiquitinating enzyme CYLD negatively regulates the ubiquitin-dependent kinase Tak 1 and prevents abnormal $\mathrm{T}$ cell responses. J Exp Med 204: 1475-1485, 2007.

16 Wang L, Baiocchi RA, Pal S, Mosialos G, Caligiuri M and Sif S: The BRG1- and hBRM-associated factor BAF57 induces apoptosis by stimulating expression of the cylindromatosis tumor suppressor gene. Mol Cell Biol 25: 7953-7965, 2005.

17 Wo L, Lu D and Gu X: Knockdown of miR-182 promotes apoptosis via regulating RIP1 deubiquitination in TNF-alphatreated triple-negative breast cancer cells. Tumour biology : the journal of the International Society for Oncodevelopmental Biology and Medicine 37: 13733-13742, 2016.

18 Ni F, Gui Z, Guo Q, Hu Z, Wang X, Chen D and Wang S: Down-regulation of miR-362-5p inhibits proliferation, migration and invasion of human breast cancer MCF7 cells. Oncology letters 11: 1155-1160, 2016.

19 Chen CA and Okayama H: Calcium phosphate-mediated gene transfer: a highly efficient transfection system for stably transforming cells with plasmid DNA. Biotechniques 6: 632-638, 1988.

20 Hollestelle A, Nagel JH, Smid M, Lam S, Elstrodt F, Wasielewski M, Ng SS, French PJ, Peeters JK, Rozendaal MJ, Riaz M, Koopman DG, Ten Hagen TL, de Leeuw BH, Zwarthoff EC, Teunisse A, van der Spek PJ, Klijn JG, Dinjens WN, Ethier SP, Clevers H, Jochemsen AG, den Bakker MA, Foekens JA, Martens JW and Schutte M: Distinct gene mutation profiles among luminal-type and basal-type breast cancer cell lines. Breast Cancer Res Treat 121: 53-64, 2010.

Received April 6, 2017

Revised May 16, 2017

Accepted May 24, 2017 